\title{
Building an Architectural Component Model for a Telehealth Service
}

\author{
Alan Taylor, Greg Morris, Jennifer Tieman, David Currow, Michael Kidd, Colin Carati \\ Faculty of Medicine, Nursing and Health Sciences, Flinders University, Adelaide, Australia \\ Email: alan.taylor@flinders.edu.au
}

Received 11 August 2015; accepted 11 September 2015; published 16 September 2015

Copyright $@ 2015$ by authors and Scientific Research Publishing Inc.

This work is licensed under the Creative Commons Attribution International License (CC BY).

http://creativecommons.org/licenses/by/4.0/

cc) (i)

Open Access

\begin{abstract}
Models of services, processes and technology are useful tools for conceptualizing complex systems such as healthcare. The application of a component architecture helps illustrate the processes and technologies that are important to the operation of a health service and conceptualize the relationships between each component. Telehealth services are relatively recent and have characteristics that do not fit neatly into established models of health services. This paper analyzes the components used to build a telehealth in the home service in South Australia and the design choices that were taken. The service used commodity-based devices and systems to deliver simple to use, low-cost in the home care. Building on this analysis, the components required in an architectural component model of a telehealth service are identified enabling a provisional architecture for telehealth services to be derived from an existing internationally recognized architectural model for eHealth systems. Situated within the broad family of eHealth architectures, a Telehealth Architectural Model of telehealth processes, software, devices, common systems and ICT infrastructure is proposed that represents the components required to support telehealth and allows for customization of services according to clinical models of care.
\end{abstract}

\section{Keywords}

Architectural Models, eHealth, Telehealth in the Home, Healthcare Processes, Infrastructure, Infostructure, Governance

\section{Introduction}

Over 2013-2014, Flinders University and the Southern Adelaide Local Health Network (SALHN) of South Australian Health (SA Health) collaborated to develop a telehealth service as part of a trial called "Flinders Telehealth in the Home: Palliative and Aged Care in SA South Australia” (FTH trial), which is funded through the 
Australian Government Department of Health. The FTH trial was an action-research initiative evaluating the use of video conferencing alongside other applications and services for use by hospital-based community outreach services. The participating services shared key characteristics: existing community clinical outreach, specialists with a desire to work more closely with general practitioners and a need to cover a large geographic region. The trial introduced telehealth services in three clinical areas:

1. Community based palliative care. Patients and their carers received video-conferencing and remote monitoring services from a specialist palliative care nurse using a tablet device (iPad), a self-assessment application to record their ongoing symptoms, and electronic devices and scales to monitor their physical activity and weight. General practitioners were able to participate in clinical case conferencing.

2. Home-based rehabilitation services for the elderly at home. Patients were monitored by a therapist remotely, who made video calls as required, and also had access to rehabilitation and speech therapists used a tablet device (iPad), a self-assessment application to record their health status and an exercise tracking device to monitor their physical activity [1].

3. Residential Aged Care Facilities (RACFs). Geriatricians used video-conferencing to residents and nurses to provide aged care assessments, plus advice on rehabilitation and medications.

Clinical care was delivered from the Repatriation General Hospital in Adelaide, South Australia to FTH trial participants, using internet protocol (IP) based video conferencing. Connectivity between the hospital and participants was achieved through the Australian National Broadband Network (NBN) and mobile data services from an Australian telecommunications provider (Telstra 3G/4G). Key technical components of the FTH trial included devices, communications, applications and management systems. Components were built using consumer technology obtained from a range of providers, with different levels of service provision, performance or availability.

This paper evaluates the applicability of an existing internationally recognized architectural model for eHealth systems to this type of telehealth in the home services. Models of services, processes and technology are useful tools for conceptualizing complex systems such as healthcare. Application of a component architecture helps to illustrate the processes and technologies that are important to the operation of a health service and conceptualize the relationships between each component. The major component categories of this model are foundation infrastructure, infrastructure, healthcare processes and governance. Through identification of the constituent parts of each category, this paper derives a provisional architecture for telehealth services.

Ethics approval for the trial was obtained through the Southern Adelaide Clinical Human Research Ethics Committee, application numbers HREC/13/SAC/88(168.13 and 203.13).

\section{2. eHealth Systems Modeling}

\subsection{An eHealth Architecture Model}

The International Standards Organisation has proposed an eHealth architecture model (eHAM) [2] that provides a generalized component architecture view of eHealth systems. A simplified version of this framework is shown in Figure 1. The intention of this framework is to assist countries and organizations achieve the improvements and benefits shown in the pyramid (apex at the top of the model) - i.e. improved quality of and access to care, improved efficiency, productivity and cost-effectiveness, informed health policy and increased evidence-based practice. The components in the model are intended to be typical, optional and configurable to meet local needs when being used in any specific eHealth service. As such, this model provides the basis for the development of further architectural models within an eHealth family of patient records, image management, mobile health and telehealth applications. This model is used as a template within which to describe the management and technical components of FTH trial.

The eHealth architecture model comprises different component categories that are aggregated under the following general categories:

- Foundation component-ICT infrastructure-is shown as the bottom layer of Figure 1, and covers the core IT technologies including networking, servers, software and IT human resources. Foundation components are underpinned by relevant standards, methods, guidelines and frameworks.

- Foundation component-infostructure-is shown as the middle layer of Figure 1, and includes electronic health record repositories, registries, data interchange interoperability, consent and access control, privacy and security and data warehousing. 


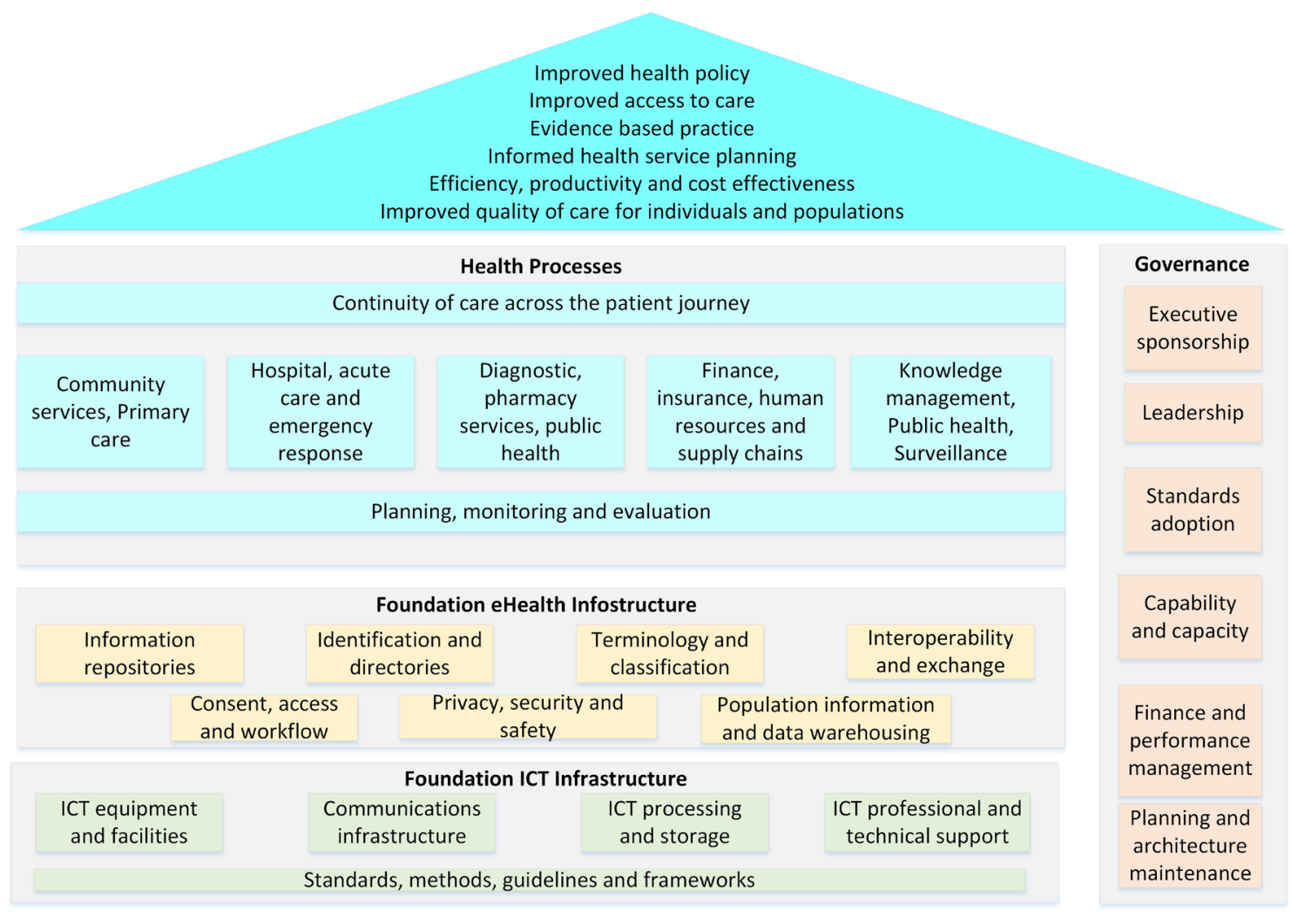

Figure 1. eHealth architecture model (eHAM) simplified (ISO/TR 14639-2:2014).

- Health process domain component is shown as the upper layer of Figure 1. These processes involve patients seeking and accessing healthcare services and providers offering these services. Health domains include a broad range of services such as clinical (provider) assessment of health problems, diagnostic (test) assessments, therapeutics and related components, such as payment for services and evaluation of services, provider and patient education and knowledge management which span the continuum of care.

- Governance and ownership is shown as the vertical bar on the far right of Figure 1. Governance and ownership are an important category of the eHealth architecture model since it represents the organizational and governance aspects of eHealth, including the financing, performance management and the development of local capability and capacity in health informatics. Standards are a key contributor to both infrastructure and infostructure.

\subsection{Business Requirements}

The FTH trial rehabilitation and palliative care clinical teams provided the business requirements against which each component of the FTH trial was assessed. Requirements focused on the need to provide solutions appropriate and affordable to the over 65 age group of people, some of whom are frail. Consequently the foundation eHealth infrastructure and infostructure components were, as far as possible, designed to conform to the following requirements:

- usability—easy for clinicians and patients to use;

- cost and compatibility — built with compatible consumer grade applications and equipment;

- interoperability - interoperable with existing health sector ICT infrastructure;

- performance - can sustain adequate video and audio quality over a variety of networks; and

- privacy—can protect the privacy of patients.

\subsection{Assessment of Architectural Models}

Assessment of architectural models is a recent development. In Australia the National eHealth Transition Au- 
thority has developed a set of eHealth Architecture Principles [3] that can be applied to eHealth initiatives. These principles provide statements and rationale for a number of qualities important in healthcare, such as quality, safety, interoperability, efficiency, security, reuse of assets etc. In other domains, such as software engineering, a number of methodologies have been developed to evaluate architecture [4], that also consider qualities derived from business requirements such as interoperability and extensibility.

\subsection{From eHealth to Telehealth Architecture}

This paper describes the architectural components used in the FTH trial, and the extent to which they met business requirements using the general categories provided in the eHAM, namely: Infrastructure, infostructure, health processes and governance. Through this process the key components relevant to telehealth services are identified for inclusion within a provisional telehealth architectural model.

\section{Foundation ICT Infrastructure}

\subsection{Devices}

Consumer tablet devices for patients were considered from the outset to be the preferred option for the patient hardware platform that would provide a simple to use, low-cost solution. It was the intention of the trial to develop applications that would work on the two major tablet platforms (Android and Apple IOS). Devices for rehabilitation and palliative care patients and clinicians were purchased from local retailers. Software updates were obtained over the internet from suppliers. For example iPads received regular operating system updates from Apple. iPads were specifically customized with a different set of applications for rehabilitation and palliative care patients and clinicians. Detailed assessment of available tablets in the retail market was based on the following requirements:

- accessibility of on/off power buttons and easy to attach power connectors for people who may have limited dexterity;

- availability of good quality stands;

- availability of 1280 by 720 pixel front-facing camera;

- usability of the interface and reasonable size screen for people who may have poor eyesight;

- pricing in the retail market at an affordable level.

Of the available devices, the Apple iPad and the Samsung Galaxy TAB 3 (Android) tablets provided the above features. The pricing of the Apple iPad on the retail market was at the higher end of tablet devices while the Android tablet was at the lower end of the market. Compatibility with applications for both these platforms was and continues to be a problem. Several video conferencing applications were available for Android or iPad devices but not both platforms. The Apple iPad was the preferred hardware platform for the clinical services involved in the trial, so it was chosen for the FTH trial.

\subsection{Communications, Processing and Storage}

Flinders University provided the majority of the underpinning organizational ICT services and was the focal point for the network architecture (Figure 2). These included local area network access (fixed and wireless), telephony, mobile phones, computer desktops, operating systems, desktop applications, email, printing, processing (for applications) storage (documents and email) and network security.

Originally, the trial was designed to operate over the National Broadband Network (NBN) being implemented across Australia from 2014. This network provided "fibre to the home" connections, with minimum bandwidths of $25 \mathrm{Mbps}$ up/5 Mbps down. Delays in the rollout of the network, and the need for rapid connections for clients, resulted in the use of commercial wireless networks.

Rehabilitation and palliative care participants at home were provided with an Apple iPad with WiFi connectivity, and a NBN "ready" router with inbuilt WiFi. Where NBN connectivity was not available in a location, iPads with Telstra 3G/4G capabilities were loaned to participants to take home on discharge from hospital. The networking required in the home was quite simple; the primary device being used was an iPad with WiFi connectivity, and a router with inbuilt wireless IEEE $802.11 \mathrm{n}$ provided connection to the internet. An NBN connection with a speed of $25 \mathrm{Mbps} / 5 \mathrm{Mbps}$ was utilized as this allowed for the highest quality of video conferencing available on the video conferencing client. 


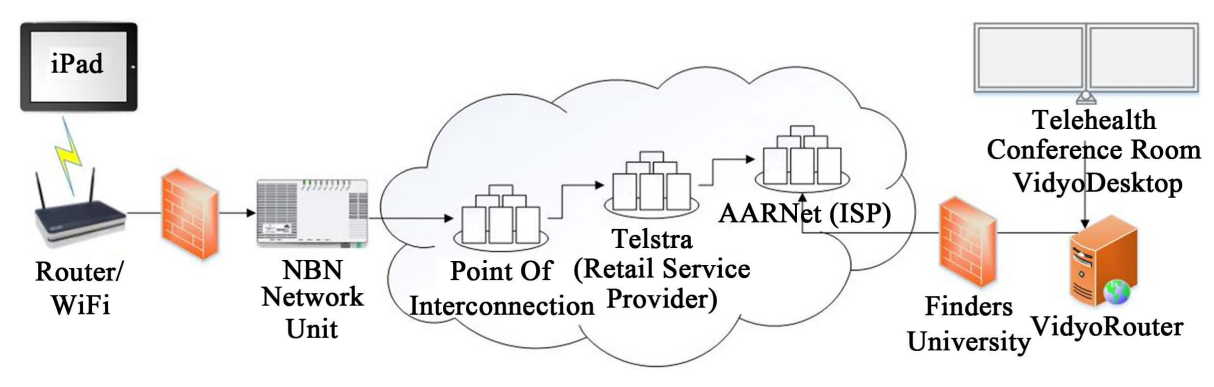

Figure 2. Network architecture.

The deployment of iPads with mobile data capabilities, negated the need for any equipment in the home. This proved to be a successful "easy-to-deploy" method for the tele-rehabilitation team, although the palliative care team needed to use a WiFi access point to provide connectivity to Fitbit Aria [5] scales which require a WiFi network to connect to the Fitbit servers. We chose to implement a combined USB + WiFi modem, providing mobile broadband connectivity for both the iPad and Fitbit Aria scales. Both arrangements enabled clinical staff members to implement a home telehealth service without the need for ICT staff to attend the patients' homes.

\subsection{Professional and Technical Support}

Flinders University provided core professional and technical support. This included, project management, technical, administrative support and evaluation expertise. Specific technologies such as video conferencing and some applications were supported under agreements with suppliers. Since the majority of the equipment and many applications were purchased from retailers or online, support for these was minimal.

Technical support staff provided application development, research and high level problem-solving, including the development of applications, training materials and guides. These staff configured patient iPads and managed online or telecommunications services. They resolved issues and tested hardware, software, video conferencing, connectivity issues, internet and telecommunications services. The technical team was tightly integrated with clinical teams on the premise that a collaborative approach between clinical and ICT specialists would contribute to rapid technology innovation and better support of clinical service needs.

\section{Foundation eHealth Infostructure Systems-Video Conferencing}

The eHAM infostructure component focuses on systems that support health records such as repositories. Telehealth services require a different component set. One such component is a video conferencing system. Video conferencing access was provided to all clinicians and patients on desktop and mobile devices. The FTH trial aimed to provide video conference systems compatible with existing SA Health and private health care providers, since care provision for the participating clinical teams crosses all these sectors. On grounds of cost and complexity (an additional device in the home), the use of hardware-based video products was not considered. The assessment criteria for software based video conferencing solutions included:

- $\quad$ ease of use, functionality and usability of the management platform;

- adequate video and audio quality over a variety of networks;

- secure/encrypted connection and a contact list that permits patient privacy;

- interoperability with standard video conferencing protocols;

- based on international standards, reducing the risk of product lock-in to one supplier;

- availability for major mobile platforms with support available locally from the vendor;

- cost and licensing arrangements.

It was thought from the outset that elderly patients may not have the technical knowledge to navigate through complex interfaces and procedures. Therefore video conferencing clients were assessed to determine if patients were required to:

- enter login credentials each time the app was opened or device was rebooted;

- enter a call or meeting ID to join conference;

- navigate interfaces containing too many options/buttons (as found in most collaborative based software) or not professional in appearance (often due to a social media focus). 
The video conferencing application used by clinicians and FTH trial participants was based on a proprietary system (Vidyo) [6] which was chosen following an initial evaluation of several providers. Consumer video services such as Skype or Apple Facetime were not adopted after consideration of the required usability in clinical settings, performance, privacy and interoperability. Vidyo interoperability with video conferencing standard protocols was provided through a gateway. Field tests confirmed that Vidyo was the preferred choice of clinical staff.

During evaluation of the product, a critical risk to patient confidentiality was discovered. The Vidyo system is structured around video room tenancies. Vidyo allows any user within a tenancy to call another member in the same tenancy. While the chances of a patient being able to navigate the system and dial a random contact number were minimal, it was still considered to be a critical risk to patient privacy and needed to be addressed. Therefore a multi-tenant Vidyo environment was implemented to maintain patient privacy as an on-premise, Flinders University hosted solution. The clinicians' tenancy allowed visibility of all patient tenancies, providing them the ability to view a patient's online status and to dial out to the patients.

\section{Health Services Software and Devices}

\subsection{Clinical Software}

SA Health provided FTH trial clinical staff with access to patient admission information, scheduling of clinics and an electronic medical record system for use during telehealth consultations. However, additional applications specific to each clinical discipline were required. The following clinical applications were made available to patients on an iPad tablet, and clinicians on a computer or iPad tablet:

- palliative care support, monitoring and assessment tools provided through the Care Search [5] website;

- prescribed rehabilitation exercises through a cloud-hosted application (TRex) [7] that provides rehabilitation patients with a personalized list exercises;

- exercise monitoring via a cloud-hosted application synchronized to the exercise monitoring device, for both palliative care and rehabilitation patients; and

- consumer rehabilitation "apps" available "off the shelf” through the Apple App store.

The rehabilitation care model required the exercise application be managed remotely, allowing for the telerehabilitation team to update a patient's exercise plan as they progressed with their recovery. The application replaced the current practice of giving patients a printout each time an exercise was added or removed from that patient's treatment. Other requirements included usability, ease of navigation, minimal patient input, ability for clinician to update remotely, content appropriate for target audience, and video with voiceover for each exercise. An application was developed based on a managed cloud appliance using entirely open-source software. An open-source platform was chosen that provided a low-cost, device agnostic and rapidly scalable service.

Therapeutic consumer applications for iPads, Android tablets and Windows tablets were assessed, with a view to providing them to patients to encourage faster recovery. Therapeutic activity does not replace a customized rehabilitation program devised by trained rehabilitation professionals but supports people who do not have access to continuing rehabilitation services.

Criteria for selecting applications for their therapeutic benefit were established. The selected applications [8] were sorted by: price (free or low cost); therapeutic content; area of the body they act on; and the device (iPad, android/windows tablet) they can be used on.

The need to schedule a large number of carers required a patient-centered scheduling application. For Telehealth in the Home services, the primary function of a scheduling application was to act as an organizer and memory aid to assist a client with a cognitive disability to manage telehealth appointments, tasks and activities in daily life in their own home, with the assistance of a scheduler (therapist, family member).

\subsection{Clinical Devices}

Many medical and monitoring devices used in healthcare are specific to a discipline. In the FTH trial the Fitbit@zip activity monitoring devices for patients were provided to both rehabilitation and palliative care patients. The Fitbit@zip is a device available in the retail market and assisted in the analysis of patient movement and weight data. The Fitbit@zip records steps taken and was chosen for its suitability to the needs of this group of rehabilitation and palliative care patients, its long battery life and the ability to upload the recorded data to a remote server for analysis. However palliative care clinicians found that this device had limited usefulness for 
some patients who were notable to move around very quickly, as the device failed to register their low activity patterns. In contrast rehabilitation patients were able to use the device to set exercise targets, thereby increasing activity.

\section{Healthcare Processes}

\subsection{Clinical Care}

Rehabilitation and palliative care disciplines have very different models of care. Palliative care required a low touch, non-intrusive approach for checking on the health of patients at home over a lengthy period. Rehabilitation care tried to provide the greatest impact in the shortest amount of time so that patients recovered within a four to six week treatment period. Rehabilitation scheduled frequent video conferencing and exercise applications while palliative care relied on less frequent contact but greater remote assessment of patients. Frequent interactions between rehabilitation clinicians and patients were supported by the scheduling application.

\subsection{Planning, Monitoring and Evaluation}

The FTH trial was based on action research methods and had robust planning, monitoring and evaluation targets set by the funding body. Many health organizations struggle to fund ongoing evaluation of services, but in the case of the FTH trial it was important to evaluate the acceptability and efficiency of a new service. It was shown that palliative care, rehabilitation and aged care services delivered by telehealth can:

- support consumers in collaborating with and communicating with their carers and health services;

- identify health issues earlier and provide an increased intensity of care;

- $\quad$ increase the accessibility of services and reduce travel for clinicians and patients;

- reduce clinical service costs;

- $\quad$ successfully deploy simple consumer grade technology to patients and clinicians.

\section{Governance}

\subsection{Sponsorship and Leadership}

Governance of the FTH trial was provided by a steering group that included clinical and technical leads. On a day-to-day basis the FTH trial manager provided finance and contract control, advice on business requirements, system architecture and the use of guidelines and standards. Clinical leadership from each of the rehabilitation and palliative care services was crucial to the implementation of telehealth technology within the clinical care models.

\subsection{Service Management}

Deploying consumer devices and applications configuration becomes a significant problem as patient numbers increase because many applications rely on email details linked to a personal password. This problem was partially overcome by linking application configuration to devices, which in turn were assigned to individuals. Consequently, a robust asset and configuration system was required. By applying unique asset numbers to ICT equipment, staff could easily identify which asset had been assigned to each patient and clinician. The FTH trial relied on two applications to deploy and manage iPads for patients and clinical staff members:

- Apple Configurator; an application designed by Apple for easy deployment of iOS devices within an organization [9]. The Apple configurator allowed "imaging" of an iPad to a standard "base image".

- Meraki; an internet cloud based mobile device manager that is hosted free of charge by Cisco [10]. It allows for easy management of iOS settings, policies, and application.

The FTH trial originally used spreadsheet software to track assets. However as asset numbers grew, it became clear that a more sophisticated system was needed. A custom designed database was built to record and track assets including: asset number, serial number, manufacturer, type of equipment, IMEI device number, mobile broadband SIM service number and asset allocation.

\subsection{Security and Access Management}

Research protocols require patient identifiers to be used to keep the identities of patients anonymous to non- 
clinical or non-SA Health staff. The identity management system used a unique asset number assigned to patient devices. The unique asset number was the only identifier used to authenticate users to the ICT systems.

\subsection{Finance and Performance Management and Capability}

Each of the technical solutions implemented in the trial were able to scale substantially at a low incremental cost. The trial implemented a number of open-source, highly-scalable technology services allowing for rapid growth in telehealth, while maintaining a low ongoing maintenance and hosting cost. The exercise application and selfassessment tools can scale tenfold with no increase in operating costs. The video conferencing application was the least scalable of all of the technologies implemented, due to the costs of increasing the number of licenses for additional lines to provide additional video conferencing capacity.

The sustainability analysis of the FTH trial found that health system costs during the trial reduced by about $50 \%$, and would improve even more once patient volumes exceeded a few hundred per annum. It was recommended that telehealth services to the home should focus on hospital avoidance, increasing access to disadvantaged groups, and being able to meet projected rising demand within existing resources. Since Australia has a health system funding model in which community, primary and hospital services receive funding from different bodies, necessary first steps in widespread implementation of telehealth to the home will be the definition of responsibilities, funding, payments for patients with severe conditions likely to attend hospital, and primary/community care responsibilities for illness prevention, and stable chronic disease management.

\subsection{Standards, Guidelines, Quality and Safety}

Several Australian organizations have produced telehealth guidelines [11]-[13]. The South Australian Health Department (SA Health) developed Guidelines for Sub-acute Services offering Digital Telehealth Network Consultations [14] between practitioners and patients in hospital settings. The FTH trial was also able to apply some of the guidelines in the recently published International Standards Organisation Telehealth Guidelines specification (ISO/TS 13131) [15] [16].

\section{A Telehealth Architecture Model}

The eHAM model is further developed to better represent telehealth services inthe FTH trial. The resulting architectural model is customized for telehealth services in the form ofa provisional Telehealth Architectural Model (THAM) containing:

- A governance category applicable to all the components on the left hand side of the model including sponsorship, service management, security and access, finance and performance, capability and capacity, business requirements, standards, guidelines, quality and safety.

- An infostructure category that takes account of the need to customize software and devices to support specific models of clinical care. These clinically specific applications remain dependent on systems such as health records and in the case of telehealth, video conferencing. By highlighting the importance of discipline specific processes and applications, the THAM model acknowledges that the processes being used in health care are increasingly codified in applications and devices.

- Foundation infrastructure that supports health processes, software, systems and devices comprises common facilities (accommodation, devices, computers, tablets), communications infrastructure (mobile data and internet services), processing, storage and support services. Increasingly this infrastructure also relies on commodity devices, networks and services such as cloud services.

From the standpoint of a patient focused telehealth in the home service it is worth noting that the eHAM architecture model only portrays a high level view of the health system as understood by health care organizations, and does not illustrate the roles of clinical services and patients. The THAM model in Figure 3 therefore recognizes that health services provide care to people in the community. While it is possible to compare the eHAM and THAM models, it should be noted that the International Standards Organisation eHealth architecture model is intended as a guide for healthcare organizations and clinical services to develop architectures customized for the specific services they provide.

A limitation of this type of architectural model is that it is essentially static. Community characteristics, needs of patients, the response of health services to those needs and the technologies used may change over time. This 


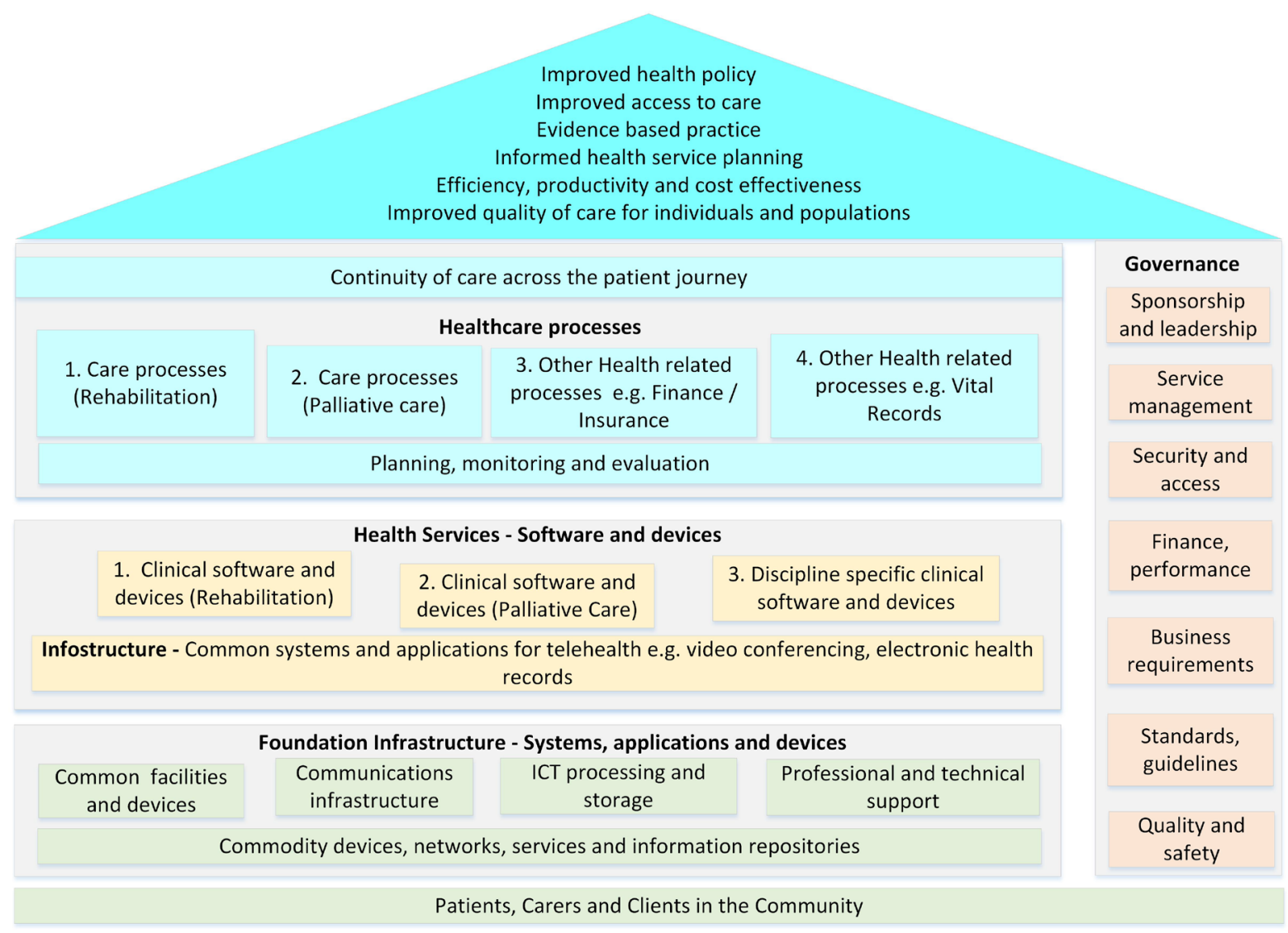

Figure 3. FTH trial TeleHealth architecture model (THAM).

issue is worthy of further research. The benefits of an architectural view of telehealth services, processes, components, activities and policies are that a systematic approach can be taken to the building of investments in ICT to support delivery of healthcare services.

\section{Conclusions}

This paper shows that telehealth to the home care depends on common (foundation) components, applications and devices, but that it is necessary to customize components to support the specific requirements of each clinical service. Successful implementation of a telehealth in the home service also depends on sponsorship by clinicians, development of a service management capability, provision of appropriate technology capacity, security (and access) management and access to standards or guidelines.

The FTH trial demonstrates that low-end consumer technology can support clinical care. Technical solutions evolve rapidly, leading to new options for models of care that may be more efficient. However, design and management of the technical ecosystem is complex and requires careful consideration of usability, scalability and interoperability among devices, applications and systems.

A Telehealth Architecture Model (THAM) as a derivative of the more generalized eHealth Architectural Model (eHAM) takes account of the complexity of telehealth systems in an era of widespread consumer-based internet services. THAM represents the components required to support telehealth and allows for customization of services according to clinical models of care. It may be of value to apply the THAM model to a large number of clinical settings that require health care to be delivered across multiple settings of care, including care for people at home.

\section{Acknowledgements}

The FTH trial was an initiative funded by the Australian Government. The authors would also like to thank all 
participants in the FTH trial, staff at the Repatriation General Hospital in Adelaide and fellow researchers including Professor Maria Crotty, Kate Swetenham, and Dr. Craig Whitehead.

\section{Declaration of Conflicting Interests}

The authors declare that there are no conflicts of interest.

\section{References}

[1] Crotty, M., Killington, M., van den Berg, M., Morris, C., Taylor, A. and Carati, C. (2014) Telerehabilitation for Older People Using Off-the-Shelf Applications: Acceptability and Feasibility. Journal of Telemedicine and Telecare, 20, 370-376. http://dx.doi.org/10.1177/1357633X14552382

[2] ISO/TR 14639-2 (2014) Health Informatics-Capacity-Based eHealth Architecture Roadmap-Part 2: Architectural Components and Maturity Model. International Standards Organisation, Geneva.

http://www.iso.org/iso/catalogue_detail?csnumber=54903

[3] NEHTA (2012) eHealth Architecture Principles. Version 1.0, National E-Health Transition Authority, Sydney. https://www.nehta.gov.au/implementation-resources/ehealth-foundations/interoperability-framework

[4] Ionita, M., Hammer, D. and Obbink, H. (2002) Scenario-Based Software Architecture Evaluation Methods: An Overview. Workshop on Methods and Techniques for Software Architecture Review and Assessment at the International Conference on Software Engineering, Orlando, 19-24 2002, 2-12.

[5] CareSearch, Palliative Care Knowledge Network. http://www.caresearch.com.au/Caresearch/Default.aspx

[6] Frost \& Sullivan Global Video Conferencing Technology Leadership Award White Paper. http://info.vidyo.com/WP-2013-Global-Video-Conferencing-Technology-Leadership-Award.html?ls=White\%20Paper

[7] Flinders University of South Australia. Telerehab. http://www.flinders.edu.au/mnhs/telehealth/resources/telehealth-rehab-exercises-trex.cfm

[8] Flinders University of South Australia. Therapeutic Apps. http://www.flinders.edu.au/mnhs/telehealth/resources/therapeutic-apps.cfm

[9] Apple Configurator. https://itunes.apple.com/au/app/apple-configurator/id434433123?mt=12

[10] Meraki Systems Manager. https://meraki.cisco.com/products/systems-manager

[11] Australian College of Rural and Remote Medicine. ACRRM Telehealth Guidelines. http://www.ehealth.acrrm.org.au/system/files/private/ACRRM\%20Telehealth\%20Guidelines_v1.0_20120827.pdf

[12] Australian Health Practitioner Regulation Agency. Guidelines for Technology Based Patient Consultations. http://www.medicalboard.gov.au/Codes-Guidelines-Policies.aspx

[13] Royal Australian College of General Practitioners. Guidelines for Inter-Professional Collaboration between General Practitioners and Other Medical Specialists Providing Video Consultations. http://www.racgp.org.au/your-practice/guidelines/interprofessional/

[14] South Australian Health. Guidelines for Sub-Acute Services Offering Digital Telehealth Network Consultations. http://www.sahealth.sa.gov.au/wps/wcm/connect/public+content/sa+health+internet/health+reform/clinical+networks/r ehabilitation+clinical+network

[15] ISO/TS 13131 (2014) Health Informatics_-Telehealth Services-Quality Planning Guidelines. International Standards Organisation, Geneva. http://www.iso.org/iso/home/store/catalogue_tc/catalogue_detail.htm?csnumber=53052

[16] Taylor, A. (2015) Applying International Guidelines for Telehealth Services-A Case Study. Proceedings of the 8th Australasian Workshop on Health Informatics and Knowledge Management, Sydney, 27-30 January 2015, 87-95. 